\title{
SINGLE MOLECULE SPECTROSCOPY OF ELECTRON TRANSFER
}

\author{
Michael Holman, Ling Zang, Ruchuan Liu, David M. Adams \\ Department of Chemistry, Columbia University \\ New York, New York 10027
}

The objectives of this research are threefold: (1) to develop methods for the study electron transfer processes at the single molecule level, (2) to develop a series of modifiable and structurally well defined molecular and nanoparticle systems suitable for detailed single molecule/particle and bulk spectroscopic investigation, (3) to relate experiment to theory in order to elucidate the dependence of electron transfer processes on molecular and electronic structure, coupling and reorganization energies. We have begun the systematic development of single molecule spectroscopy (SMS) of electron transfer and summaries of recent studies are shown below.

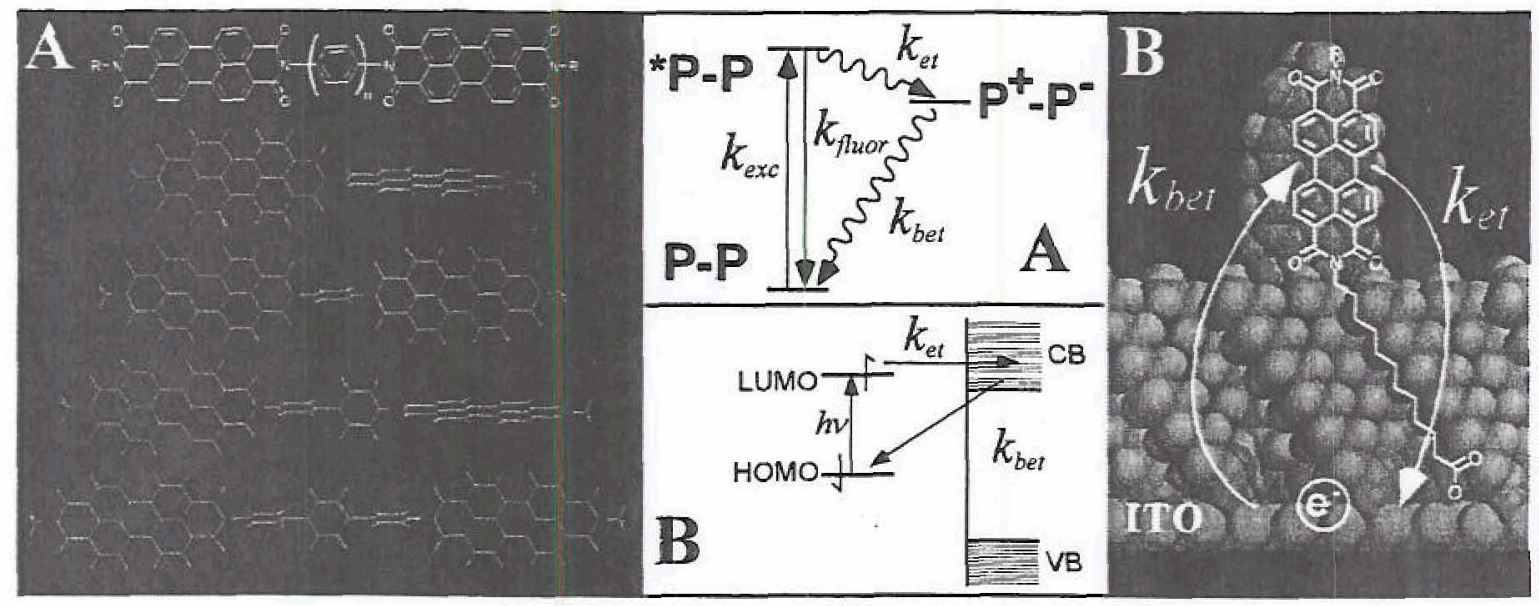

There is a tremendous need for experiments designed to probe the discrete electronic and molecular dynamic fluctuations of single molecules near electrodes and at nanoparticle surfaces. Single molecule spectroscopy (SMS) has emerged as a powerful method to measure properties of individual molecules which would normally be obscured in an ensemble-averaged measurement. Fluctuations in the fluorescence time trajectories contain detailed molecular level statistical and dynamical information of the system. The full distribution of a molecular property is revealed in the stochastic fluctuations, giving information about the range of possible behaviors that lead to the ensemble average. In the case of electron transfer, this level of understanding is particularly important to the field of molecular and nanoscale electronics: from a device-design standpoint, understanding and controlling this picture of the overall range of possible behaviors will likely prove to be as important as designing in the ideal behavior of any given molecule.

Domor-Bridge-Acceptor Systems. We have used SMS to investigate intramolecular electron transfer (IET) processes in a series of model dimer systems composed of two perylene chromophores connected via an adjustable bridge (Fig A). 
The fluorescence behaviors of a large number of molecules were cataloged and the results statistically analyzed to gauge information about the range of behaviors of the ensemble. Single-molecule fluorescence time trajectories reveal "blinks", momentary losses in fluorescence ( $>20 \mathrm{~ms}$ to seconds in duration), which are attributed to discrete IET excursions to the charge separated (CS) state. The observed statistical trends corroborate the assignment of the blinking behavior to IET. We find that fluorescence blinking behavior is dependent on bridge length and chromophore geometry, which affect the electronic coupling and therefore the IET.

Donor-Bridge-Electrode Systems. We have used SMS to measure the rates of photoinduced interfacial electron transfer in chromophore-bridge-electrode systems composed of perylene molecules in mixed self-assembled monolayers on ITO semiconductor electrodes (Fig. B). We have observed that the fluorescence of single perylene molecules in these structures display blinking behavior with characteristic millisecond off periods. These off periods are ascribed to discrete ET events - the molecule is not fluorescent in the charge transfer state. The fluorescence blinking is not seen for molecules on polymer coated glass surfaces since there are not states available for ET (i.e. electrode states). Electron transfer rates were obtained directly from the analysis of the fluorescence time trajectories. For many molecules studied, single exponential behavior is observed for both forward and back ET processes. Variability in the observed rates is attributed to local variations in the monolayer structure.

We are presently studying at the single molecule and bulk levels, the electric field and dielectric dependence of electron transfer rates in these systems. We are also incorporating Marcus theory for electron transfer into our analysis of the experimental data and are using quantum mechanical methods to calculate the electronic structure and electron transfer coupling matrix elements. Ongoing and future work includes the extension of this methodology to study electron transfer in other molecular structures and devices and in heterodimer systems composed of single perylene molecules ${ }^{1}$ and high quality nanoparticles of $\mathrm{TiO}_{2}$.

1) We previously developed perylene chromophores that bind to oxide surfaces. See Ling Zang, Ruchuan Liu, Michael W. Holman, Kim T. Nguyen, and David M. Adams, "A Single Molecule Probe Based on Intramolecular Electron Transfer", J. Am. Chem. Soc. 2002, 124, 10640.

Publications under DOE Basic Energy Sciences Funding (2002-2003)

1) Michael W. Holman, Ruchuan Liu, and David M. Adams, "Single Molecule Spectroscopy of Interfacial Electron Transfer", submitted to J. Am. Chem. Soc.

2) Ruchuan Liu, Michael W. Holman, Ling Zang, and David M. Adams, "Single Molecule Spectroscopy of Intramolecular Electron Transfer in Donor-BridgeAcceptor Systems", submitted to J. Phys. Chem. 